\title{
Design of Cost-effective QoL Infotainment System
}

\section{Arunava Bhattacharya, Jabeena A.}

\begin{abstract}
In vehicle infotainment (IVI) systems are becoming increasingly popular in vehicles. It is less a modern luxury and more a utility as the quality of life $(Q o L)$ of the average individual improves. The aim of this project is to attempt a more efficient design with low cost hardware that improves the Quality of Life (QoL) of the driver and passengers of a vehicle. The method of design is to use commonly available resources and appropriately interfacing them to create an infotainment system that collects, displays and stores information. The collection of information is local, the display is both local and over a cloud, and the storage is over a cloud. The data collected by the sensors could also be processed according to a manufacturer at either the microcontroller, or the phone application, and relevant information can be displayed on an attached screen and on the cloud for analysis by a manufacturer.
\end{abstract}

Keywords - QoL, IVI, sensors, cloud

Table of Contents:

\begin{tabular}{|c|c|c|}
\hline Introduction & & 1 \\
Fig. 1 & Schematic & 2 \\
& & \\
Results & & 2 \\
& & \\
Fig. 2 & App. - Not Connected & 2 \\
Fig. 3 & App. - Connected & 2 \\
Fig. 4 & App Inv. Backend & 2 \\
Fig. 5 & Acc. Y axis & 3 \\
Fig. 6 & Acc. X axis & 3 \\
Fig. 7 & Acc. Z axis & 3 \\
Fig. 8 & LDR graph & 3 \\
Fig. 9 & Humidity graph & 4 \\
Fig. 10 & Temperature graph & 4 \\
Fig. 11 & Gas graph & 4 \\
& & \\
Cost Analysis & & 4 \\
and & & \\
Conclusion & & \\
& & \\
Acknowledgements & & \\
References & & \\
& & \\
\end{tabular}

I. INTRODUCTION

The components used in the project are pretty standard components for a lot of electronic equipment one may find around oneself. This makes the components and replacements easier to procure and very affordable. Now, coming more specifically to the functions of these components: these are sensors that detect quality of air, ambient light, jerk motion, temperature and humidity within a vehicle. These parameters monitor the health of the people inside a vehicle as well as the vehicle itself.

Upon further calibrations, it would be possible to come up with formulae and expressions that can tell the End User exactly what to do based on the data the sensors are receiving. For example, air quality is degrading - open the windows; or there's been too much jerk motion within a certain period of time - the car needs maintenance.

Currently, the prototype is functional in the following segments of data collection and representation:

1. Sensors are connected to an Arduino board, and take a reading every $1100 \mathrm{~ms}$ (higher ceiling tick rate that all sensors work at)

2. Arduino sends the data to a Bluetooth module wirelessly connected to a smartphone.

3. This smartphone has an application that receives the data in a prescribed text format and arranges them in a list by splitting the received text at every prescribed delimiter for an End User to read. It also sends the data corresponding at every 15 seconds' interval to an open source cloud.

4. The cloud stores the data and represents it in a graphical format which would make it easier for an individual to analyse it.
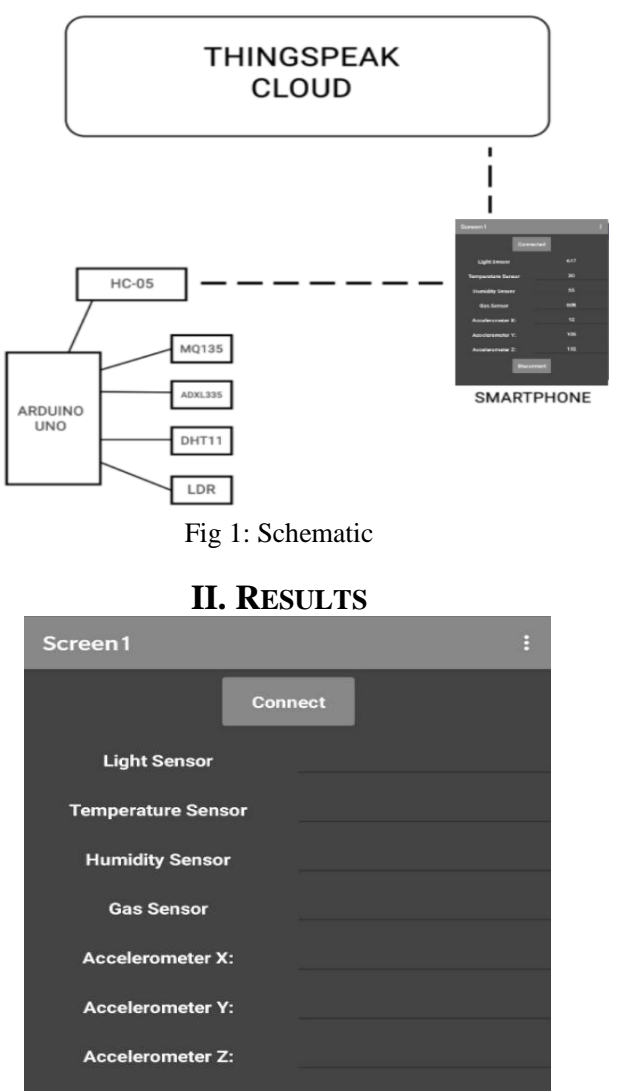

Fig 2: Not Connected

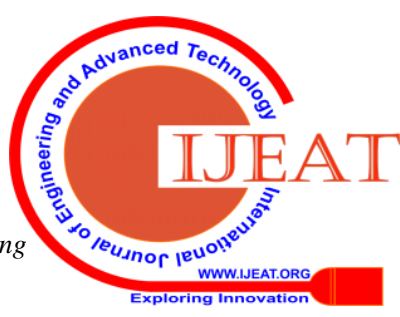




\section{Design of Cost-effective QoL Infotainment System}

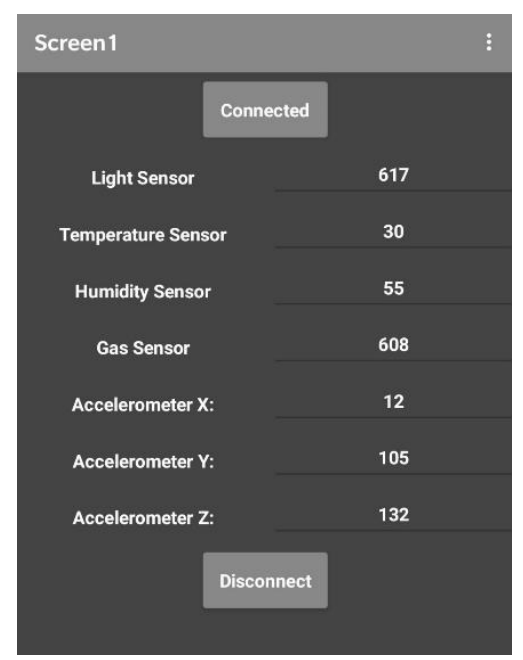

Fig 3: Connected

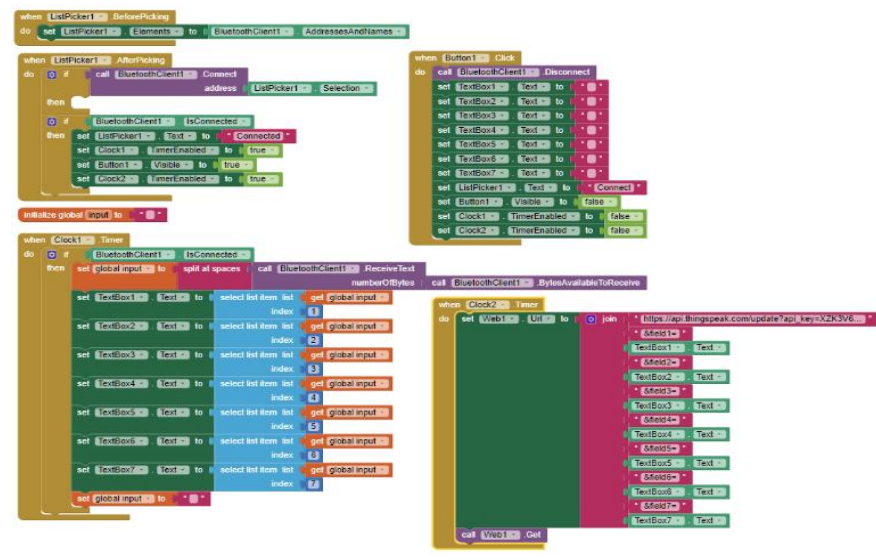

Fig 4: Function Blocks

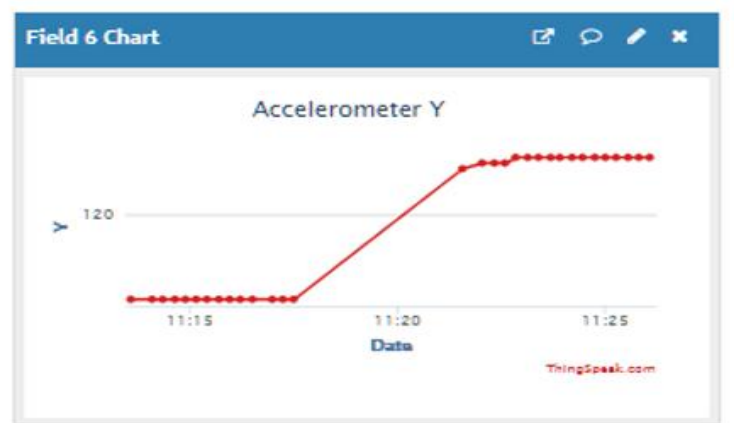

Fig 5: This graph represents the values the ADXL335 accelerometer's $Y$ axis sensor sends to the Arduino\}

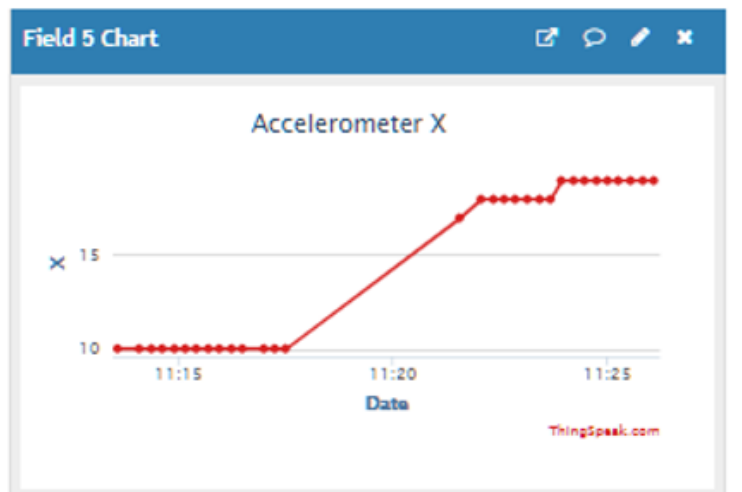

Fig 6: Ihis graph represents the values the AUXL335 accelerometer's $X$ axis sensor sends to the Arduino.

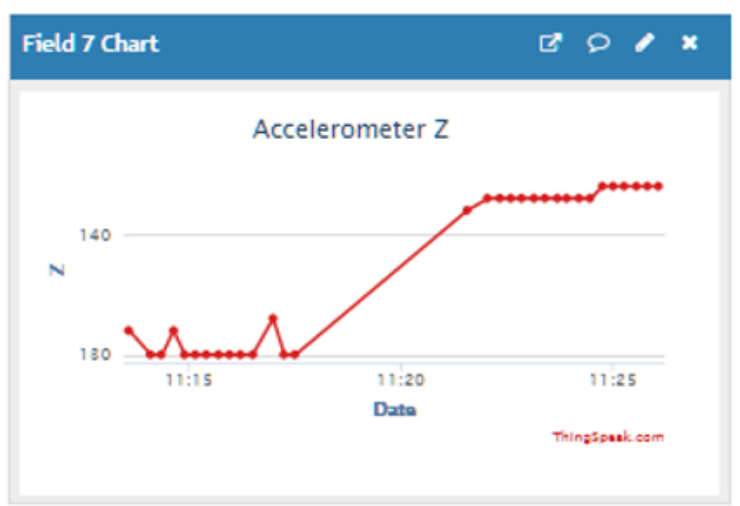

Fig 7: This graph represents the values the ADXL335 accelerometer's $Z$ axis sensor sends to the Arduino.

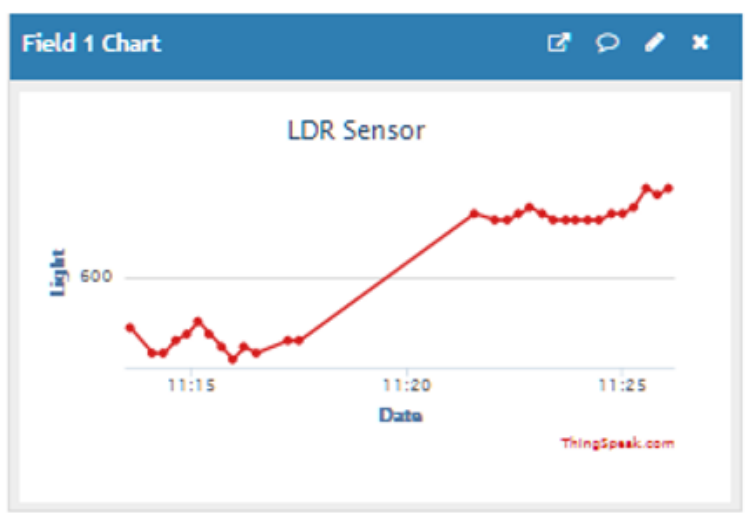

Fig 8: This graph represents the values the LDR Light illuminance sensor sends to the Arduino.

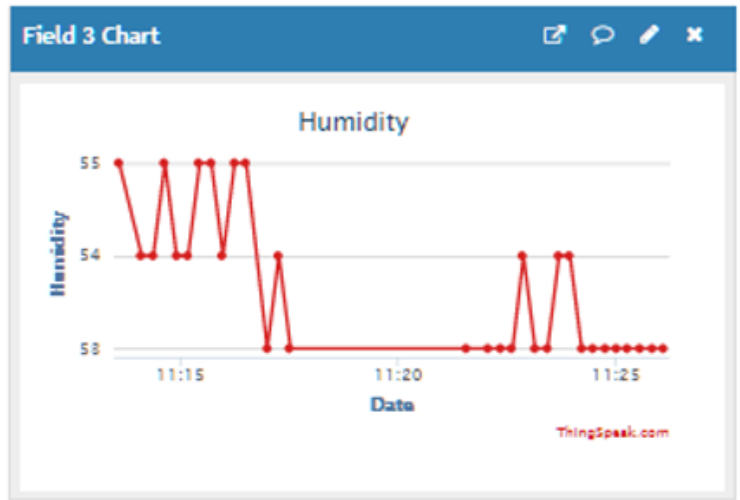

Fig 9: This graph represents the values the DHT11 humidity sensor sends to the Arduino.

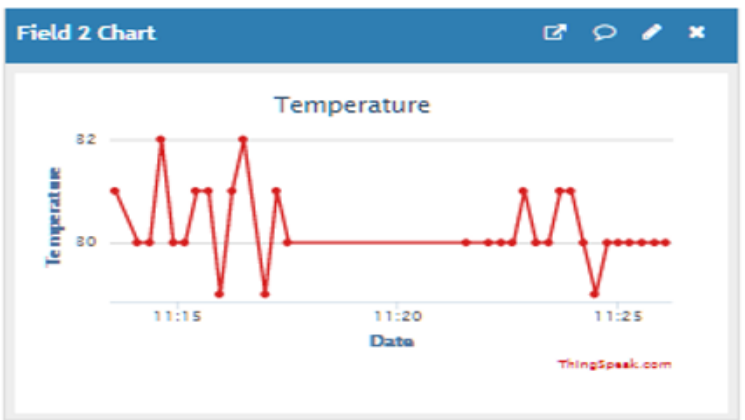

Fig 10: This graph represents the values the DHT11 temperature sensor sends to the Arduino.

Published By:

Blue Eyes Intelligence Engineering

\& Sciences Publication

(C) Copyright: All rights reserved.

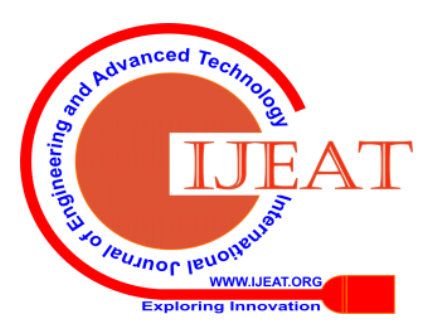




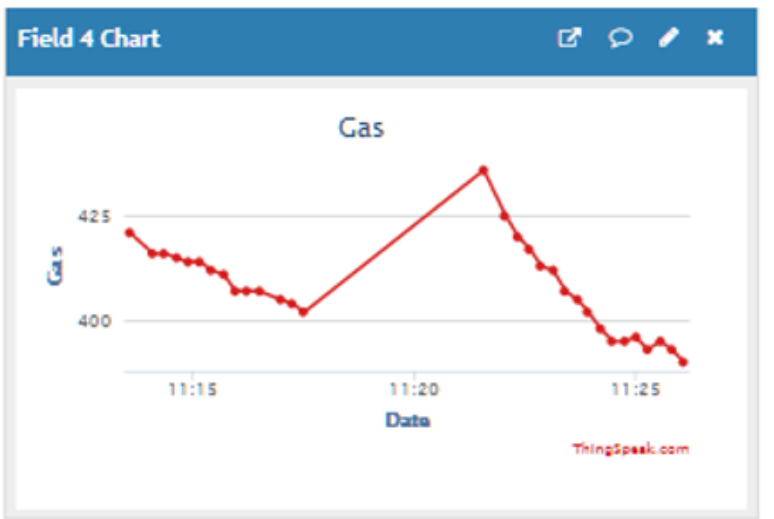

Fig 11: This graph represents the values the MQ135 gas sensor sends to the Arduino.

\section{COST ANALYSIS AND CONCLUSIONS}

- 4 sensors: Rs. 1200

- Arduino: Rs. 500

- $\quad$ BT module: Rs. 300

- $\quad$ Power Source

- $\quad$ Screen: Rs. 1000 or Mobile Device

Total Cost Estimate: Min. Rs. 3000.

The prototype functions as expected. The physical compactness of the device can be improved. Bluetooth module is a little unstable resulting in occasional packet loss. The mobile application needs more improvement over handling packet loss: it just shows an index mismatch error at every occurrence.

\section{ACKNOWLEDGEMENTS}

This project has given me a treasure of knowledge and experience that I believe I would find essential in all my future endeavours. I express my gratitude to my guide for her constant and meticulous judgement in improving my project, and in turn me. I would like to thank my institute, VIT Vellore, for providing me an opportunity to carry this project to fruition.

\section{REFERENCES:}

1. Miguel Galarza , Josep Paradells: 'Improving road safety and user experience by employing dynamic in-vehicle information systems', IET Intell. Transp. Syst., 2019, Vol. 13 Iss. 4, pp. 738-744

2. Sahil Garg, Kuljeet Kaur, Syed Hassan Ahmed, Abbas Bradai, Georges Kaddoum, Mohammed Atiquzzaman: 'MobQoS: MobilityAware and QoS-Driven SDN Framework foa Autonomous Vehicle', IEEE Wireless Communications, August 2019, Vol. 26 Iss. 4, pp. 1220

3. Yunke Wang, Xiaoming Liu: 'An In-Vehicle Infotainment Test Data Management Method based on XML Schema', Chinese Control And Decision Conference, June 2019, IEEE September 2019

4. S.M. Ahsan Kazmi, Tri Nguyen Dang, Ibrar Yaqoob, Anselme Ndikumana, Ejaz Ahmed, Rasheed Hussain, and Choong Seon Hong: 'Infotainment Enabled Smart Cars: A Joint Communication, Caching and Computation Approach', IEEE Transactions on Vehicular Technology, Vol. 68 , Iss. 9 , Sept. 2019

5. Dong-Kyu Choi, Joong-Hwa Jung, Seok-Joo Koh, Ji-In Kim, Juyoung Park: 'InVehicle Infotainment Management System in Internet-ofThings Networks', International Conference on Information Networking, Jan. 2019, IEEE May 2019

6. Hangsik Shin: 'Ambient temperature effect on pulse rate variability as an alternative to heart rate variability in young adult', 2016, J Clin Monit Comput 30, 939-948
Published By: Blue Eyes Intelligence Engineering DOI: 10.35940/ijeat.E9844.069520

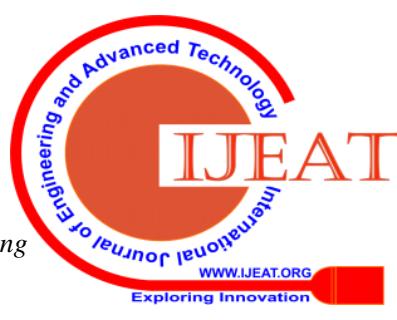

